\title{
Improving Visual Sensitivity with Subthreshold Transcranial Magnetic Stimulation
}

\author{
Arman Abrahamyan, ${ }^{1}$ Colin W. G. Clifford, ${ }^{1}$ Ehsan Arabzadeh, ${ }^{2}$ and Justin A. Harris ${ }^{1}$ \\ ${ }^{1}$ School of Psychology, University of Sydney, New South Wales 2006, Australia, and ${ }^{2}$ School of Psychology, University of New South Wales, New South \\ Wales 2052, Australia
}

We probed for improvement of visual sensitivity in human participants using transcranial magnetic stimulation (TMS). Stimulation of visual cortex can induce an illusory visual percept known as a phosphene. It is known that TMS, delivered at intensities above the threshold to induce phosphenes, impairs the detection of visual stimuli. We investigated how the detection of a simple visual stimulus is affected by TMS applied to visual cortex at or below the phosphene threshold. Participants performed the detection task while the contrast of the visual stimulus was varied from trial to trial according to an adaptive staircase procedure. Detection of the stimulus was enhanced when a single pulse of TMS was delivered to the contralateral visual cortex 100 or $120 \mathrm{~ms}$ after stimulus onset at intensities just below the phosphene threshold. No improvement in visual sensitivity was observed when TMS was applied to the visual cortex in the opposite hemisphere (ipsilateral to the visual stimulus). We conclude that TMS-induced neuronal activity can sum with stimulus-evoked activity to augment visual perception.

\section{Introduction}

Transcranial magnetic stimulation (TMS) is a noninvasive technique for stimulating a target brain area via electromagnetic induction using a coil placed next to the scalp (Barker et al., 1985a; Hallett, 2007; O'Shea and Walsh, 2007; Wagner et al., 2009). TMS of the occipital lobe excites cortical neurons (Moliadze et al., 2003) to create a retinotopically localized illusory visual percept known as a phosphene (Barker et al., 1985b; Meyer et al., 1991). Many studies have shown that TMS, at intensities above the threshold to induce phosphenes, interferes with normal visual processing and impairs the detection of visual stimuli (Amassian et al., 1989; Kammer, 2007; Harris et al., 2008). Here we examined the effect of stimulation at or below the phosphene threshold on the detection of visual stimuli.

We hypothesized that TMS-induced excitation of visual neurons would sum with the excitation of those neurons by a visual stimulus presented within their receptive field. As a consequence, TMS would augment the strength of the visual percept. A simple way to test this hypothesis is to combine a weak TMS pulse to visual cortex with a weak visual stimulus, such that the summed neural response would be above the detection threshold, even though both individual inputs are below that threshold. Put differently, if we measure how strong a visual stimulus must be before an observer can detect it (i.e., the observer's visual threshold), that threshold should be reduced if the stimulus is accompanied by low-intensity TMS to the visual cortex.

In three experiments, participants initially received TMS pulses to the occipital lobe to identify the most suitable stimula-

\footnotetext{
Received Dec. 1, 2010; revised Jan. 6, 2011; accepted Jan. 13, 2011.

This research was funded by the Australian Research Council Grant DP0986137.

Correspondence should be addressed to Justin A. Harris, School of Psychology, University of Sydney, NSW 2006, Australia. E-mail: justin.harris@sydney.edu.au.

DOI:10.1523/JNEUROSCI.6256-10.2011

Copyright $\odot 2011$ the authors $\quad 0270-6474 / 11 / 313290-05 \$ 15.00 / 0$
}

tion site that reliably evokes phosphenes (Cowey and Walsh, 2000). We then measured each participant's phosphene threshold. Next, the participants performed a behavioral task to detect a plaid pattern presented on a monitor while receiving single pulses of TMS (see Fig. 1). The position of the plaid was aligned to the apparent location of the phosphenes, as established at the start of testing, so that the plaid was located within the receptive field of the neurons targeted by TMS. The participants' detection thresholds for the plaid were measured using an adaptive staircase procedure that varied the contrast of the visual stimulus using a Bayesian algorithm (Kontsevich and Tyler, 1999) that maximizes the expected information gain from each trial. Our aim was to measure how detection thresholds for the plaid varied as a function of the intensity of TMS delivered to the visual cortex.

\section{Materials and Methods}

\section{Participants}

We tested a total of 17 volunteers, including the authors, who had normal or corrected-to-normal vision (9 participants were male; mean age, 31 years; age range, 22-43 years). Experimental procedures were approved by the Human Research Ethics Committee at the University of Sydney.

\section{Visual stimulus}

The visual stimulus (Fig. 1) was a plaid pattern constructed of two superimposed sinusoidal gratings oriented at $+45^{\circ}$ and $-45^{\circ}$. The sinusoidal gratings had a spatial frequency of 1 cycle per degree and were multiplied by a symmetric two-dimensional Gaussian contrast envelope to eliminate sharp edges (peak contrast $=100 \%$; full width at half-height $=2.2^{\circ}$ of visual angle). The stimulus was presented within a black circular ring that subtended $6.5^{\circ}$ of visual angle against a uniform gray background of $77 \mathrm{~cd} / \mathrm{m}^{2}$ luminance.

\section{Equipment}

Visual stimuli were presented on a 19 inch cathode ray tube monitor (P992, BenQ) operating at a $75 \mathrm{~Hz}$ refresh rate. Gamma correction was applied to ensure a linear luminance characteristic. The participant's 


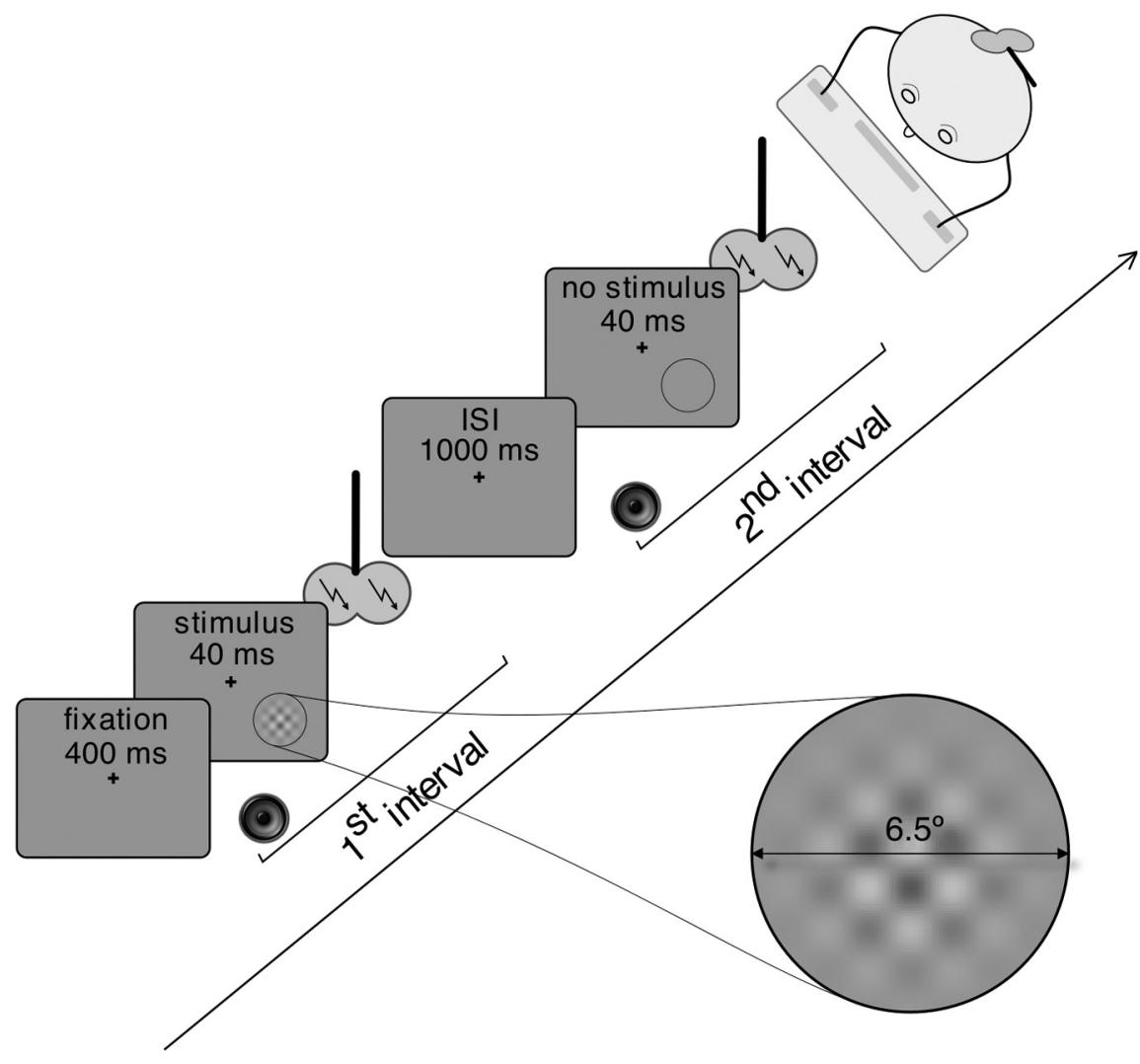

Figure 1. Experiment design. In three experiments, we tested participants on a two-interval, two-alternative forced-choice task. A visual plaid stimulus was presented for $40 \mathrm{~ms}$ in the middle of one of two sequential $1 \mathrm{~s}$ intervals (first or second interval, with equal probability). The start of each interval was signaled by a brief auditory cue. Participants had to fixate the cross in the center of the monitor and report which interval contained the visual stimulus by pressing one of two keys. During both intervals, a single pulse of TMS was delivered either to the occipital lobe (experimental conditions) or to the (z site located on the top of the head at the intersection of the midline and interaural line (control conditions).

head was supported by chin and forehead rests at a viewing distance of 57 $\mathrm{cm}$ from the monitor. Stimulus presentation and synchronous triggering of the TMS pulse were programmed in Matlab (MathWorks) assisted by Psychtoolbox (Brainard, 1997; Pelli, 1997).

We delivered TMS using a Magstim Rapid ${ }^{2}$ system (Whitland) with a $70 \mathrm{~mm}$ figure-eight coil held by an adjustable articulated arm (Manfrotto). The position of the coil was guided using Softaxic neuronavigation system (EMS Medical).

\section{Procedure}

TMS protocol. Participants sat in a dimly lit room and dark adapted for $\sim 5 \mathrm{~min}$. The TMS coil was positioned with the handle oriented horizontally pointing to the left of the participant and tangential to the scalp. The coil was initially placed against the back of the participant's head, with the center over an area $3 \mathrm{~cm}$ above the inion and $2 \mathrm{~cm}$ lateral. Single pulses were delivered with intensities reaching $80 \%$ of the stimulator's output, while the coil was moved in steps of $0.5-1 \mathrm{~cm}$. The position of the coil that evoked bright phosphenes located in the lower contralateral visual field away from the fovea was marked as a "hotspot." The coil was fixed in this position using an articulated arm, and the neuronavigation system was used to track online the coil position relative to the participant's head to keep it within $2 \mathrm{~mm}$ of the hotspot throughout experimental procedures. Phosphenes were elicited by stimulating the left $(n=14)$ or right $(n=3)$ occipital lobe. Participants indicated the location of a phosphene by using a mouse to move a circle on the monitor while keeping fixation in the center of the monitor. The control site for TMS was $\mathrm{Cz}$, which is the point of intersection between the midline (from nasion to inion) and the interaural line. In this condition, the coil was placed horizontally on the top of the participant's head, with handle pointing backwards, and the center of the coil over Cz. As reported below, visual thresholds measured in the
$\mathrm{Cz}$ control condition did not differ from the thresholds obtained during initial psychophysical testing without TMS.

Accurate estimation of the phosphene threshold was important for this study because we expected that the improvement in visual sensitivity would occur when stimulation intensity was close to the phosphene threshold. To measure the phosphene threshold, we asked participants to close their eyes and maintain their gaze at the remembered location of the fixation point on the screen. We also asked them to ignore the loudness of the TMS click when detecting phosphenes. To estimate phosphene thresholds accurately, we developed a procedure (Abrahamyan A, Clifford CW, Ruzzoli M, Phillips D, Arabzadeh E, and Harris JA, unpublished observations) that uses a Matlab toolbox to control the stimulator output combined with a Bayesian adaptive staircase (Kontsevich and Tyler, 1999). On each trial of the 30-trial staircase, a single pulse of TMS was presented at an intensity that varied from trial to trial, and the participant reported by key press whether they saw a phosphene or not. The estimated threshold corresponded to the stimulation intensity that evokes phosphenes on $60 \%$ of trials (position parameter of a Weibull function fitted to the proportion of phosphene responses between 0 and 1 with a lapse rate of $4 \%$ ). The phosphene threshold was calculated as the mean value obtained from two or three staircases.

Visual task. We measured the participants' thresholds for detection of a plaid pattern using a two-interval, two-alternative, forcedchoice task (Fig. 1). In both intervals of each trial, a black circular ring was displayed for 40 $\mathrm{ms}$ at the previously established location of the phosphene. The plaid stimulus was presented inside the ring in one of the two intervals with equal probability. The start of each interval was signaled by a brief auditory tone, and the intervals were separated by $1 \mathrm{~s}$. Participants had to indicate by key press whether the stimulus was presented in the first or second interval. The stimulus contrast varied from trial to trial according to a Bayesian adaptive staircase (Kontsevich and Tyler, 1999). A single staircase lasted for 30 trials, at the end of which an estimate of the detection threshold was calculated, corresponding to an accuracy of $80.3 \%$ correct. We tested each participant on multiple 30-trial staircases under each condition, and staircases run under the different conditions were randomly intermixed. Thus, for each participant the visual detection threshold for each condition was calculated as an average of several independently estimated thresholds. (We ran a mean of 3.9 staircases per condition in experiment 1a, 5.1 staircases in experiment $1 \mathrm{~b}$, and 2.5 staircases in experiment 2.) Differences in the amount of testing between participants were due to differences in their availability for repeated testing.

During the visual detection task, TMS was applied either to the occipital lobe or to the $\mathrm{Cz}$ control site. On each trial, two identical pulses of TMS were delivered, one pulse in each interval. We did this so that the mere presence of the pulse could not bias the participants' decision about which interval contained the plaid pattern. The intensity of the TMS pulse to visual cortex was varied across conditions in experiments $1 \mathrm{a}$ and $1 \mathrm{~b}$, ranging from 60 to $120 \%$ of phosphene threshold (values tested were $60,75,80,85,90,95,100$, and $120 \%$ ). The TMS pulse delivered to $\mathrm{Cz}$ in the control condition was fixed at $90 \%$ of phosphene threshold. In all conditions, the TMS pulse was delivered either $100 \mathrm{~ms}$ (experiment 1a) or $120 \mathrm{~ms}$ (experiments $1 \mathrm{~b}$ and 2) after the onset of the ring and plaid pattern (or after the empty ring in the stimulus-absent interval). These latencies were chosen based on evidence that strong pulses of TMS at 
these times interfere with visual detection (Amassian et al., 1989; Kammer, 2007), and based on our preliminary study indicating that weak pulses of TMS at these times could reduce visual detection thresholds. We hypothesized that TMS at $120 \%$ of phosphene threshold would increase the threshold for detection of the visual stimulus, but that weaker pulses of TMS, at or just below the phosphene threshold, would reduce visual thresholds. Thus, we predicted a U-shape function for the relationship between TMS intensity and visual threshold.

Experiment 2 was designed to investigate the topographic specificity of the effect of TMS on visual detection. Participants received weak pulses of TMS ( $\sim 90 \%$ of phosphene threshold) $120 \mathrm{~ms}$ after the stimulus onset. They were tested under the following three separate conditions: (1) TMS was delivered to the visual cortex contralateral to the location of the visual stimulus, so that the stimulus and phosphene were colocated (as in experiments 1a and 1b); (2) TMS was delivered to the corresponding site in the other hemisphere (ipsilateral to the visual stimulus); and (3) TMS was applied to the $\mathrm{Cz}$ control site. The three conditions were randomly intermixed in different 30 -trial blocks. The intensity of TMS was $90 \%$ of phosphene threshold for four new participants; for the other five participants, who had already participated in experiment $1 \mathrm{~b}$, the intensity of TMS was set to the value that produced the greatest decrease in their threshold in that experiment (this was $95 \%$ for four participants, and $85 \%$ for one participant). We predicted that TMS would reduce visual detection thresholds, relative to the $\mathrm{Cz}$ control condition, when applied to the contralateral visual cortex but not when applied to the ipsilateral cortex.

\section{Results}

Experiment 1a: TMS at $100 \mathrm{~ms}$

The mean phosphene threshold across all participants $(n=9)$ was $57.5 \%$ (range, 50 to $70 \%$ ) of the maximum output of the TMS machine. Based on the phosphene threshold for each participant, we delivered a single pulse of TMS $100 \mathrm{~ms}$ after onset of the visual stimulus, and across conditions we varied the intensity of TMS from 60 to $120 \%$ of the phosphene threshold. The visual threshold at which the stimulus was detected varied as a function of TMS intensity, as shown in Figure $2 A$. The U-shape characteristic of this function was confirmed by testing polynomial contrasts in a repeated-measures ANOVA conducted on data from the experimental conditions (in which TMS was applied to visual cortex), which showed there to be a significant quadratic trend in threshold across TMS intensity $\left(F_{(1,8)}=12.6, p=0.007\right)$. Follow-up two-tailed $t$ tests compared the threshold in each experimental condition with that in the $\mathrm{Cz}$ control condition. They showed that the threshold was significantly reduced when TMS was delivered to the visual cortex at intensities of $80 \%\left(t_{(8)}=2.6, p=0.03\right)$ and $90 \%\left(t_{(8)}=2.2, p=0.05\right)$ of phosphene threshold. By contrast, the threshold was significantly increased when TMS was set at $120 \%$ of phosphene threshold $\left(t_{(8)}=2.7, p=0.03\right)$. The visual thresholds obtained in the $\mathrm{Cz}$ condition were not different from those obtained in a no-TMS condition $\left(t_{(8)}=1.2, p=0.28\right)$.

\section{Experiment 1b: TMS at $120 \mathrm{~ms}$}

The mean phosphene threshold across all participants $(n=10)$ was $55 \%$ (range, 47 to $65 \%$ ) of the maximum output of the TMS machine. Based on the phosphene threshold for each participant, we delivered a single pulse of TMS $120 \mathrm{~ms}$ after onset of the visual stimulus, while varying the intensity of TMS from $60 \%$ to $120 \%$ of the phosphene threshold. Once again, the visual thresholds varied as a function of TMS intensity (Fig. $2 B$ ), and the U shape of this function was confirmed by repeated-measures ANOVA that revealed a significant quadratic trend in threshold across experimental conditions $\left(F_{(1,9)}=7.7, p=0.02\right)$. Follow-up two-tailed $t$ tests comparing thresholds at each TMS intensity against the $\mathrm{Cz}$
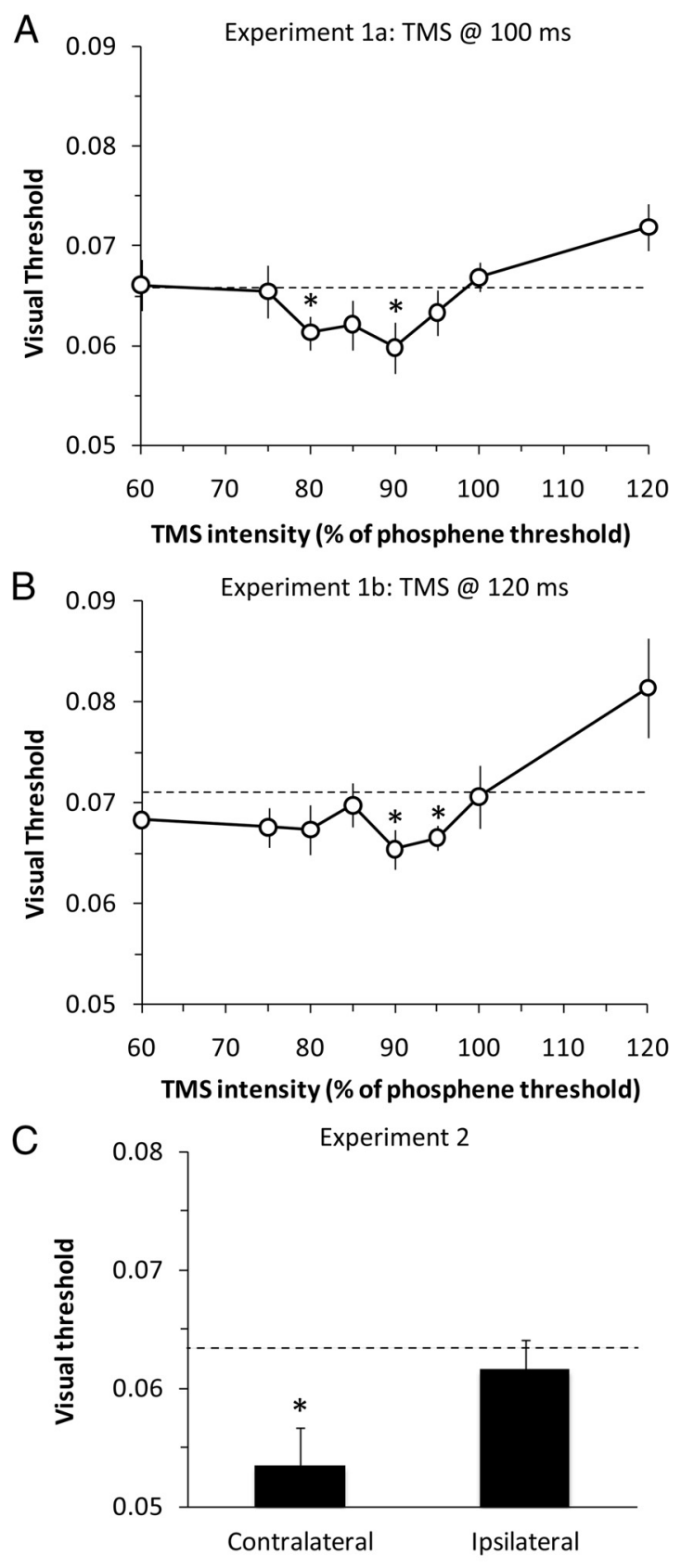

TMS location

Figure 2. $A-C$, Detection thresholds, relative to the $\mathrm{C} z$ control condition (dashed black line), in experiments $1 \mathrm{a}(\boldsymbol{A}), 1 \mathrm{~b}(\boldsymbol{B})$, and $2(\boldsymbol{C})$. Values below the dashed line indicate an improvement in visual sensitivity. In experiments $1 \mathrm{a}$ and $1 \mathrm{~b}$, a single pulse of TMS was delivered to visual cortex 100 or $120 \mathrm{~ms}$ after onset of the visual stimulus. The intensity of TMS varied from 60 to 120\% of the phosphene threshold. Experiment 2 compared the effect of TMS applied to visual cortex contralateral or ipsilateral to the stimulus, $120 \mathrm{~ms}$ after stimulus onset at an intensity just below phosphene threshold. Error bars show within-subject SEM (for the difference with $C z$ ), and asterisks identify thresholds that were significantly different from $\mathrm{Cz}(p<0.05)$.

control condition revealed that the visual threshold was significantly reduced when TMS was delivered at 90\% $\left(_{(9)}=2.9, p=\right.$ $0.02)$ and $95 \%\left(t_{(9)}=3.9, p=0.004\right)$ of phosphene threshold, but was significantly increased when TMS was delivered at $120 \%$ $\left(t_{(9)}=2.24, p=0.05\right)$. There were no differences in the visual thresholds obtained in the $\mathrm{Cz}$ condition and those obtained in a no-TMS condition $\left(t_{(9)}=1.4, p=0.20\right)$. 


\section{Experiment 2: topographic specificity}

The mean phosphene threshold across all participants $(n=9)$ was $58 \%$ (range, 48 to $70 \%$ ) of the maximum output of the TMS machine. Experiment 2 investigated whether the improvement in visual sensitivity induced by low-intensity TMS depended on delivering TMS to those cortical neurons that respond to the visual stimulus. Thus, we compared the effects of TMS delivered to the visual cortex contralateral versus ipsilateral to the visual stimulus. As shown in Figure $2 C$, visual detection thresholds were significantly lower when TMS was delivered to the contralateral visual cortex than to the ipsilateral visual cortex $\left(t_{(8)}=2.6, p=0.03\right.$, two-tailed) or to $\mathrm{Cz}\left(t_{(8)}=3.1, p=0.02\right.$, two-tailed). There was no difference between stimulation of ipsilateral visual cortex and $\mathrm{Cz}\left(t_{(8)}<1, p>0.5\right)$. Once again, there were no differences in the visual thresholds between the $\mathrm{Cz}$ condition and a no-TMS condition $\left(t_{(8)}<1, p=0.92\right)$.

\section{Discussion}

Our results demonstrate that low-intensity TMS can improve visual sensitivity. We observed a significant decrease in detection thresholds when TMS was delivered 100 or $120 \mathrm{~ms}$ after the onset of a visual stimulus when the intensity of TMS was just below the phosphene threshold. Experiment 2 additionally demonstrated that the improvement in visual sensitivity depends on stimulation of those cortical neurons whose receptive fields are occupied by the visual stimulus, since there was no improvement when TMS was delivered to the visual cortex ipsilateral to the visual stimulus. These findings show that excitation of visual cortical neurons by TMS can sum with the neuronal activity evoked by a visual stimulus to augment visual perception.

The novel finding we report here is very different from the conventional use of TMS to interfere with normal functioning of a targeted brain region (Amassian et al., 1989; Kammer, 2007; Harris et al., 2008). Indeed, experiments $1 \mathrm{a}$ and $1 \mathrm{~b}$ in the present series confirmed that, at higher intensities, TMS interferes with visual sensitivity. How then can we reconcile these opposite effects of TMS? We contend that both the improvement and impairment can be explained by a single mechanism that attributes the opposite effects of TMS to a nonlinearity in sensory processing. The explanation is based on psychophysical (Solomon, 2009) and neurophysiological (Crowder et al., 2006) evidence of a sigmoid relationship between the intensity of sensory stimulation and the magnitude of the perceptual or neural response (Fig. $3 A$ ). The key characteristic of this sigmoid function is its double inflection, with an initial accelerating component followed by a decelerating component. This characteristic is evident in the "dipper" function (Fig. 3B) that is obtained when mapping out the smallest perceptible change in stimulus intensity [the "justnoticeable difference" (JND)] as a function of the base intensity of the stimuli (Nachmias and Sansbury, 1974; Arabzadeh et al., 2008; Solomon, 2009). Thus, the biphasic nature of the present results can be understood as conforming to a dipper function, in which TMS-induced excitation of sensory neurons serves as a "pedestal" to elevate sensory activity. When TMS is applied at low intensity, sensory activity is increased only slightly and is thus shifted toward the accelerating portion of the sigmoid sensoryresponse function (Fig. 3A), which corresponds to the dip in detection threshold (Fig. $3 B$ ). When TMS is applied at higher intensity, above that sufficient to induce a visual percept (a phosphene), sensory activity is shifted beyond the dip and into the decelerating region of the response function where discrimination thresholds increase. In effect, weak pulses of TMS bring the level of activity in the sensory neurons closer to their threshold
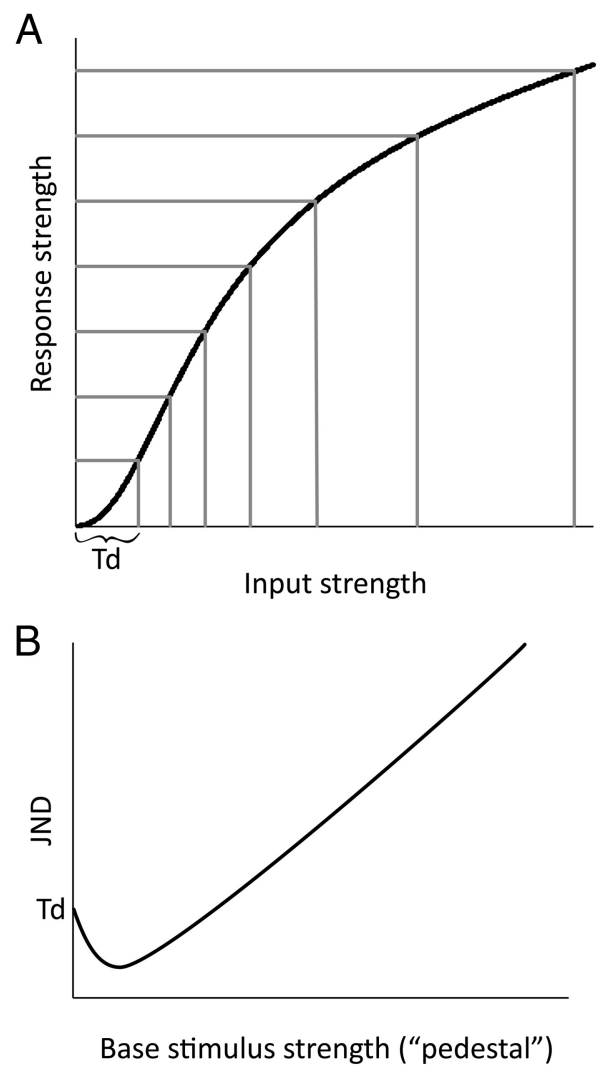

Figure 3. A illustrates a nonlinear relationship between input strength (stimulation intensity) and response strength in a sensory system. The horizontal gridlines mark the smallest resolvable units of response strength (that are perceptually discriminable); the spacing between the vertical gridlines shows the smallest detectable change in input strength (the JND). Td marks the minimum detectable input strength (the absolute detection threshold). Psychophysical evidence that the relationship between sensory input and response strength follows a sigmoid function, as shown here, comes from demonstrations that the JND between two stimuli decreases as their base strength increases, when the base strength is small (close to $\mathrm{Td}$ ), but beyond this point the JND increases as the base strength increases, as described by Weber's Law (Solomon, 2009). The biphasic nature of this change in JND as a function of base stimulus strength produces a dipper function, as shown in $\boldsymbol{B}$.

for detection of a sensory event, whereas strong intensity TMS changes the stimulus detection to a more difficult discrimination task, in which the participant must discriminate between the stimulus plus phosphene and the phosphene alone. We stress that, according to this explanation, the impact of TMS itself on neuronal activity is assumed to be monotonic, consistent with neurophysiological and electroencephalographic measurements of the acute effects of TMS (Moliadze et al., 2003; Rosanova et al., 2009). The contrasting effects of TMS at different intensities are instead attributed to a sigmoid nonlinearity in the input response function of the sensory neurons.

Evidence for a dipper function is usually obtained when measuring sensory thresholds in the presence of a pedestal that is an exact copy of the target stimulus. However, TMS, as applied here, is unlikely to be selective in activating only those neurons that respond to the visual stimulus. In other words, it is unlikely that our effect is mediated by a TMS-induced visual percept that resembles the plaid pattern that participants were required to detect. Rather, we believe that TMS acts as a "noisy pedestal" through broadband excitation of visual channels, in much the same way that a visual stimulus with noisy spatial frequency can act as a pedestal to improve detection of a simple visual grating (Henning and Wichmann, 2007). According to this view, broad- 
band activation of cortical neurons by TMS would uniformly elevate activity across sensory channels. If this activity is lowbelow perceptual threshold-it would nonetheless bring all channels close to their threshold, thus reducing the sensory input that is required for a target stimulus to selectively activate some channels above their threshold. However, if there is strong broadband excitation of cortical neurons, such that all sensory channels are activated over their perceptual threshold, this would increase the amount of sensory input that is required before a target stimulus could be discriminated from the background.

Recent studies have also reported that TMS over sensory areas (somatosensory or visual cortex) can lead to increased sensitivity for tactile or visual stimuli (Tegenthoff et al., 2005; Waterston and Pack, 2010). However, those studies differ from the present one in several important respects. Participants in those studies received continuous trains of TMS pulses lasting up to $20 \mathrm{~min}$, before they were tested for visual or tactile sensitivity in the absence of ongoing stimulation. These "off-line" repetitive stimulation protocols would have induced relatively enduring changes in the underlying cortex (Allen et al., 2007). As such, the mechanism for this effect is likely to be different from the present one in which the acute excitation of cortical neurons by TMS summed with the excitation of those same neurons in response to a visual stimulus.

The conventional interference effects reported with TMS can arise from a variety of different mechanisms of action (e.g., Walsh and Cowey, 2000; Harris et al., 2008). Therefore, the approach we describe here, testing for the summation of TMS-induced and stimulus-evoked activity at subthreshold intensities, is an important advance in identifying a specific mechanism by which TMS can interact with neuronal function. Moreover, it introduces a new way to identify the specific stimulus attributes that are coded in neuronal populations targeted by TMS. Subthreshold TMS stimulation can thus provide a powerful new technique for functional brain mapping.

\section{References}

Allen EA, Pasley BN, Duong T, Freeman RD (2007) Transcranial magnetic stimulation elicits coupled neural and hemodynamic consequences. Science 317:1918-1921.

Amassian VE, Cracco RQ, Maccabee PJ, Cracco JB, Rudell A, Eberle L (1989) Suppression of visual perception by magnetic coil stimulation of human occipital cortex. Electroencephalogr Clin Neurophysiol 74:458-462.

Arabzadeh E, Clifford CW, Harris JA (2008) Vision merges with touch in a purely tactile discrimination. Psychol Sci 19:635-641.
Barker AT, Jalinous R, Freeston IL (1985a) Non-invasive magnetic stimulation of human motor cortex. Lancet 1:1106-1107.

Barker AT, Freeston IL, Jalinous R, Merton PA, Morton HB (1985b) Magnetic stimulation of the human brain. J Physiol (Lond) 369 [Suppl]:3P.

Brainard DH (1997) The Psychophysics Toolbox. Spat Vis 10:433-436.

Cowey A, Walsh V (2000) Magnetically induced phosphenes in sighted, blind and blindsighted observers. Neuroreport 11:3269-3273.

Crowder NA, Price NS, Hietanen MA, Dreher B, Clifford CW, Ibbotson MR (2006) Relationship between contrast adaptation and orientation tuning in V1 and V2 of cat cisual cortex. J Neurophysiol 95:271-283.

Hallett M (2007) Transcranial magnetic stimulation: a primer. Neuron 55:187-199.

Harris JA, Clifford CW, Miniussi C (2008) The functional effect of transcranial magnetic stimulation: signal suppression or neural noise generation? J Cogn Neurosci 20:734-740.

Henning GB, Wichmann FA (2007) Some observations on the pedestal effect. J Vis $7: 3$.

Kammer T (2007) Masking visual stimuli by transcranial magnetic stimulation. Psychol Res 71:659-666.

Kontsevich LL, Tyler CW (1999) Bayesian adaptive estimation of psychometric slope and threshold. Vision Res 39:2729-2737.

Meyer BU, Diehl R, Steinmetz H, Britton TC, Benecke R (1991) Magnetic stimuli applied over motor and visual cortex: influence of coil position and field polarity on motor responses, phosphenes, and eye movements. Electroencephalogr Clin Neurophysiol Suppl 43:121-134.

Moliadze V, Zhao Y, Eysel U, Funke K (2003) Effect of transcranial magnetic stimulation on single-unit activity in the cat primary visual cortex. J Physiol 553:665-679.

Nachmias J, Sansbury RV (1974) Grating contrast discrimination may be better than detection. Vision Res 14:1039-1042.

O'Shea J, Walsh V (2007) Transcranial magnetic stimulation. Curr Biol 17:R196-R199.

Pelli DG (1997) The VideoToolbox software for visual psychophysics: transforming numbers into movies. Spat Vis 10:437-442.

Rosanova M, Casali A, Bellina V, Resta F, Mariotti M, Massimini M (2009) Natural frequencies of human corticothalamic circuits. J Neurosci 29:7679-7685.

Solomon JA (2009) The history of dipper functions. Atten Percept Psychophys 71:435-443.

Tegenthoff M, Ragert P, Pleger B, Schwenkreis P, Förster AF, Nicolas V, Dinse HR (2005) Improvement of tactile discrimination performance and enlargement of cortical somatosensory maps after $5 \mathrm{~Hz}$ rTMS. PLoS Biol 3:e362.

Wagner T, Rushmore J, Eden U, Valero-Cabre A (2009) Biophysical foundations underlying TMS: setting the stage for an effective use of neurostimulation in the cognitive neurosciences. Cortex 45:1025-1034.

Walsh V, Cowey A (2000) Transcranial magnetic stimulation and cognitive neuroscience. Nat Rev Neurosci 1:73-79.

Waterston ML, Pack CC (2010) Improved discrimination of visual stimuli following repetitive transcranial magnetic stimulation. PLoS One 5:e10354. 